FACTA UNIVERSITATIS (NIŠ)

Ser. Math. Inform. Vol. 36, No 3 (2021), 669 - 683

https://doi.org/10.22190/FUMI210506050G

Original Scientific Paper

\title{
SOME VECTOR FIELDS ON THE TANGENT BUNDLE WITH A SEMI-SYMMETRIC METRIC CONNECTION
}

\author{
Aydin Gezer and Erkan Karakas \\ Ataturk University, Faculty of Science, Department of Mathematics, \\ 25240 Erzurum, Turkey
}

\begin{abstract}
Let $M$ be a (pseudo-)Riemannian manifold and $T M$ be its tangent bundle with the semi-symmetric metric connection $\bar{\nabla}$. In this paper, we examine some special vector fields, such as incompressible vector fields, harmonic vector fields, concurrent vector fields, conformal vector fields and projective vector fields on $T M$ with respect to the semi-symmetric metric connection $\bar{\nabla}$ and obtain some properties related to them. Key words: Complete lift metric, semi-symmetric metric connection, tangent bundle, vector fields.
\end{abstract}

\section{Introduction}

Friedmann and Schouten introduced the notion of a semi-symmetric linear connection on a differentiable manifold [1]. Semi-symmetric metric connections play an important role in the study of Riemannian manifolds. In [2], Hayden introduced the idea of a metric connection with torsion on a Riemannian manifold. Using Hayden's idea, Yano [6] studied a semi-symmetric metric connection on a Riemannian manifold. He proved that a Riemannian manifold endowed with the semi-symmetric metric connection has vanishing curvature tensor if and only if the Riemannian manifold is conformally flat. After that, the generalization of this result for vanishing Ricci tensor of the semi-symmetric metric connection was shown by Imai in $[3,4]$.

Received March 18, 2019. accepted May 03, 2021.

Communicated by Uday Chand De

Corresponding Author: Aydin Gezer, Ataturk University, Faculty of Science, Department of Mathematics, 25240 Erzurum, Turkey | E-mail: aydingzr@gmail.com

2010 Mathematics Subject Classification. Primary 53C07; Secondary 53A45

(C) 2021 by University of Niš, Serbia | Creative Commons License: CC BY-NC-ND 
The geometry of tangent bundle $T M$ is based on the fundamental paper of Sasaki [5] published in 1958. He used a given Riemannian metric $g$ on a differentiable manifold $M$ to construct a metric $\widetilde{g}$ on the tangent bundle $T M$ of $M$. Today this metric is called the Sasaki metric. The well-known Riemannian or pseudoRiemannian metrics on $T M$ are constructed from the Riemannian metric $g$ given on $M$ by classical lifts, such as

1. The complete lift metric or the metric $I I$;

2. The metric $I+I I$;

3. The Sasaki metric or the metric $I+I I I$;

4. The metric $I I+I I I$; where $I=g_{i j} d x^{i} d x^{j}, I I=2 g_{i j} d x^{i} \delta y^{j}, I I I=g_{i j} \delta y^{i} \delta y^{j}$ are all quadratic differential forms defined globally on the tangent bundle $T M$ over $M[8]$.

In our paper [9], we originally define a semi-symmetric metric connection on the tangent bundle equipped with complete lift metric. We compute all forms of the curvature tensors of the semi-symmetric metric connection and study their properties. Also, we have investigated conditions for the tangent bundle with this connection and the complete lift metric to be locally conformally flat. The goal of the present paper is to characterize some vector fields such as incompressible, harmonic, concurrent, conformal, projective with respect to the semi-symmetric metric connection on the tangent bundle over a Riemannian manifold.

\section{Preliminaries}

Let $M$ be an $n$-dimensional differentiable manifold and $T M$ be its tangent bundle with the natural projection $\pi: T M \longmapsto M$. Coordinate systems in $M$ are denoted by $\left(U, x^{h}\right)$, where $U$ is the coordinate neighborhood and $\left(x^{h}\right), h=1, \ldots, n$ are the coordinate functions. Let $\left(y^{h}\right)=\left(x^{\bar{h}}\right), \bar{h}=n+1, \ldots, 2 n$ be the Cartesian coordinates in each tangent space $T_{p} M$ at $p \in M$ with respect to natural basis $\left\{\left.\frac{\partial}{\partial x^{h}}\right|_{p}\right\}$, where $p$ is an arbitrary point in $U$ with local coordinates $\left(x^{h}\right)$. Then we can introduce the local coordinates $\left(x^{h}, y^{h}\right)$ on the open set $\pi^{-1}(U) \subset T M$. Here, the coordinate system of $\left(x^{h}, y^{h}\right)=\left(x^{h}, x^{\bar{h}}\right)$ is called induced coordinates on $\pi^{-1}(U)$ from $\left(U, x^{h}\right)$. In the paper, we use Einstein's convention on repeated indices.

Let $X=X^{h} \frac{\partial}{\partial x^{h}}$ be the local expression in $U$ of a vector field $X$ on $M$. Let $\nabla$ be a (torsion-free) linear connection on $M$. The vertical lift ${ }^{V} X$, the horizontal lift ${ }^{H} X$ and the complete lift ${ }^{C} X$ of $X$ are given respectively by

$$
\begin{gathered}
{ }^{V} X=X^{h} \partial_{\bar{h}}, \\
{ }^{H} X=X^{h} \partial_{h}-y^{s} \Gamma_{s k}^{h} X^{k} \partial_{\bar{h}}
\end{gathered}
$$

and

$$
{ }^{C} X=X^{h} \partial_{h}+y^{s} \partial_{s} X^{h} \partial_{\bar{h}}
$$


with respect to the induced coordinates, where $\partial_{h}=\frac{\partial}{\partial x^{h}}, \partial_{\bar{h}}=\frac{\partial}{\partial y^{h}}$ and $\Gamma_{j k}^{h}$ are the components of the connection $\nabla$.

Suppose that a $(p, q)$ tensor field $S$ on $M, q>1$, is given. We then define a $(p, q-1)$ tensor field $\gamma S$ on $T M$ by

$$
\gamma S=\left(y^{s} S_{s i_{2} \ldots i_{q}}^{j_{1} \ldots j_{p}}\right) \partial_{\overline{j_{1}}} \otimes \ldots \otimes \partial_{\overline{j_{p}}} \otimes d x^{i_{2}} \otimes \ldots \otimes d x^{i_{q}}
$$

with respect to the induced coordinates $\left(x^{i}, y^{i}\right)$ [8]. The tensor field $\gamma S$ determines a global tensor field on $T M$. We easily see that for any $(1,1)$ tensor field $P, \gamma P$ has components

$$
(\gamma P)=\left(\begin{array}{c}
0 \\
y^{j} P_{j}^{i}
\end{array}\right)
$$

and $\gamma P$ is a vertical vector field on $T M$.

With the connection $\nabla$, the set of the $2 n$ linearly independent vector fields on each induced coordinate neighbourhood $\pi^{-1}(U)$ of $T M$ which are the following forms:

$$
\begin{aligned}
& E_{j}=\partial_{j}-y^{s} \Gamma_{s j}^{h} \partial_{\bar{h}}, \\
& E_{\bar{j}}=\partial_{\bar{j}} .
\end{aligned}
$$

is a frame field [8]. We call it the adapted frame and it will be written by $\left\{E_{\beta}\right\}=$ $\left\{E_{j}, E_{\bar{j}}\right\}$. With respect to adapted frame $\left\{E_{\beta}\right\}$, the vertical lift ${ }^{V} X$, the horizontal lift ${ }^{H} X$ and the complete lift ${ }^{C} X$ of $X$ are respectively expressed by [8]

$$
\begin{aligned}
{ }^{V} X & =X^{j} E_{\bar{j}}, \\
{ }^{H} X & =X^{j} E_{j}, \\
{ }^{C} X & =X^{j} E_{j}+y^{s} \nabla_{s} X^{j} E_{\bar{j}} .
\end{aligned}
$$

The complete lift metric ${ }^{C} g$ on the tangent bundle $T M$ over a (pseudo-)Riemannian manifold $(M, g)$ is defined as follows:

$$
\begin{aligned}
{ }^{C} g\left({ }^{H} X,{ }^{H} Y\right) & =0 \\
{ }^{C} g\left({ }^{H} X,{ }^{V} Y\right) & ={ }^{C} g\left({ }^{V} X,{ }^{H} Y\right)=g(X, Y), \\
{ }^{C} g\left({ }^{V} X,{ }^{V} Y\right) & =0
\end{aligned}
$$

for all vector fields $X$ and $Y$ on $M$ [8]. Note that ${ }^{C} g$ is a pseudo-Riemannian metric on $T M$. The covariant and contravariant components of the complete lift metric ${ }^{C} g$ on $T M$ are respectively given in the adapted local frame by

$$
{ }^{C} g_{\alpha \beta}=\left(\begin{array}{cc}
0 & g_{i j} \\
g_{i j} & 0
\end{array}\right)
$$


and

$$
{ }^{C} g^{\alpha \beta}=\left(\begin{array}{cc}
0 & g^{i j} \\
g^{i j} & 0
\end{array}\right)
$$

The semi-symmetric metric connection $\bar{\nabla}$ on $T M$ with respect to the complete lift metric ${ }^{C} g$ is given as follows.

Proposition 2.1. [9]The semi-symmetric metric connection $\bar{\nabla}$ on the tangent bundle TM with the complete lift metric ${ }^{C} g$ over a (pseudo-)Riemannian manifold $(M, g)$ is given by

$$
\left\{\begin{array}{l}
\bar{\nabla}_{E_{i}} E_{j}=\Gamma_{i j}^{k} E_{k}+\left\{y^{s} R_{s i j}^{k}+y_{j} \delta_{i}^{k}-y^{k} g_{i j}\right\} E_{\bar{k}}, \\
\bar{\nabla}_{E_{i}} E_{\bar{j}}=\Gamma_{i j}^{k} E_{\bar{k}} \\
\bar{\nabla}_{E_{\bar{i}}} E_{j}=0, \bar{\nabla}_{E_{\bar{i}}} E_{\bar{j}}=0
\end{array}\right.
$$

with respect to the adapted frame $\left\{E_{\beta}\right\}$, where $\Gamma_{i j}^{h}$ and $R_{h j i}^{s}$ respectively denote components of the Levi-Civita connection $\nabla$ and the Riemannian curvature tensor field $R$ of the pseudo-Riemannian metric $g$ on $M$.

\section{Some Vector Fields on $T M$ with respect to Semi-symmetric Metric Connection}

In this section, we firstly search the properties of being harmonic and incompresible of the lifting vector fields. After that we will find the general forms of concurrent, conformal, projective vector fields with respect to the semi-symmetric metric connection on the tangent bundle $T M$ and give some important results related to them.

\subsection{Lifting vector fields being incompressible (divergence-free) and harmonic}

Firstly, we shall give the definition of an incompressible vector field on $T M$ with respect to the semi-symmetric metric connection.

Definition 3.1. Let $M$ be a (pseudo-)Riemannian manifold and $T M$ be its tangent bundle with the semi-symmetric metric connection $\bar{\nabla}$. A vector field $\widetilde{V}=$ $v^{h} E_{h}+v^{\bar{h}} E_{\bar{h}}$ on $T M$ is called incompressible vector field with respect to the semisymmetric metric connection if $\widetilde{V}$ satisfies the following condition

$$
\operatorname{trace}(\bar{\nabla} \widetilde{V})=\bar{\nabla}_{\alpha} \widetilde{V}^{\alpha}=0 .
$$

Proposition 3.1. Let $M$ be a (pseudo-)Riemannian manifold and TM be its tangent bundle with the semi-symmetric metric connection $\bar{\nabla}$. Then, for any vector field $V$ on $M$, 
i) The vertical lift ${ }^{V} V$ is an incompressible vector field on $T M$ with respect to the semi-symmetric metric connection $\bar{\nabla}$;

ii) The horizontal lift ${ }^{H} V$ or the complete lift ${ }^{C} V$ is an incompressible vector field on TM with respect to the semi-symmetric metric connection $\bar{\nabla}$ if and only if the vector field $V$ is incompressible on $M$ with respect to the Levi-Civita connection $\nabla$.

Proof. Using (2.1) and (2.2), we calculate

$$
\begin{aligned}
\operatorname{trace}\left(\bar{\nabla}^{V} V\right) & =\bar{\nabla}_{\alpha}{ }^{V} V^{\alpha}=\bar{\nabla}_{\bar{h}} v^{h}=0 \\
\operatorname{trace}\left(\bar{\nabla}^{H} V\right) & =\bar{\nabla}_{\alpha}{ }^{H} V^{\alpha}=\bar{\nabla}_{h} v^{h} \\
& =\left(\partial_{h}-y^{s} \Gamma_{s h}^{m} \partial_{m}\right) v^{h}+\bar{\Gamma}_{h m}^{h} v^{m} \\
& =\nabla_{h} v^{h}=\operatorname{trace}(\nabla V) \\
\operatorname{trace}\left(\bar{\nabla}^{C} V\right) & =\bar{\nabla}_{\alpha}{ }^{C} V^{\alpha}=\bar{\nabla}_{h} v^{h}+\bar{\nabla}_{\bar{h}} v^{\bar{h}} \\
& =\left(\partial_{h}-y^{s} \Gamma_{s h}^{m} \partial_{m}\right) v^{h}+\bar{\Gamma}_{h m}^{h} v^{m}+\partial_{\bar{h}}\left(y^{s} \nabla_{s} v^{h}\right) \\
& =2 \nabla_{h} v^{h}=2 \operatorname{trace}(\nabla V)
\end{aligned}
$$

from which, it is easy to see that the results (i) and (ii).

Definition 3.2. Let $M$ be a (pseudo-)Riemannian manifold and $T M$ be its tangent bundle with the semi-symmetric metric connection $\bar{\nabla}$. A vector field $\widetilde{V}=$ $v^{h} E_{h}+v^{\bar{h}} E_{\bar{h}}$ on $T M$ is called a harmonic vector field with respect to the semisymmetric metric connection $\bar{\nabla}$ if $\widetilde{V}$ satisfies the following condition

$$
\left(\bar{\nabla}_{i} \widetilde{V}^{\epsilon}\right){ }^{C} g_{\epsilon j}-\left(\bar{\nabla}_{j} \widetilde{V}^{\epsilon}\right)^{C} g_{\epsilon i}=0,
$$

where ${ }^{C} g_{i j}$ are the components of the complete lift metric ${ }^{C} g$ on $T M$.

The following lemma comes immediate from standard calculations.

Lemma 3.1. Let $M$ be a (pseudo-)Riemannian manifold and $T M$ be its tangent bundle with the semi-symmetric metric connection $\bar{\nabla}$. Then

i) For the vertical lift ${ }^{V} V$, we get

$$
\left(\bar{\nabla}_{\alpha}{ }^{V} V^{\epsilon}\right)^{C} g_{\epsilon \beta}-\left(\bar{\nabla}_{\beta}^{V} V^{\epsilon}\right)^{C} g_{\epsilon \alpha}=\left(\begin{array}{cc}
\nabla_{i} v_{j}-\nabla_{j} v_{i} & 0 \\
0 & 0
\end{array}\right) ;
$$

ii) For the horizontal lift ${ }^{H} X$, we get

$$
\begin{aligned}
& \left(\bar{\nabla}_{\alpha}{ }^{H} V^{\epsilon}\right)^{C} g_{\epsilon \beta}-\left(\bar{\nabla}_{\beta}{ }^{H} V^{\epsilon}\right){ }^{C} g_{\epsilon \alpha} \\
= & \left(\begin{array}{cc}
y^{s}\left[R_{s i a j}-R_{s j a i}+g_{s i} g_{j a}-g_{s j} g_{i a}\right] v^{a} & \nabla_{i} v_{j} \\
-\nabla_{j} v_{i} & 0
\end{array}\right) ;
\end{aligned}
$$


iii) For the complete lift ${ }^{C} V$, we get

$$
\begin{aligned}
& \left(\bar{\nabla}_{\alpha}{ }^{C} V^{\epsilon}\right){ }^{C} g_{\epsilon \beta}-\left(\bar{\nabla}_{\beta}{ }^{C} V^{\epsilon}\right){ }^{C} g_{\epsilon \alpha} \\
= & \left(\begin{array}{cc}
y^{s}\left[\nabla_{s}\left(\nabla_{i} v_{j}-\nabla_{j} v_{i}\right)+\left(g_{s i} g_{j a}-g_{s j} g_{i a}\right) v^{a}\right] & \nabla_{i} v_{j}-\nabla_{j} v_{i} \\
\nabla_{i} v_{j}-\nabla_{j} v_{i} & 0
\end{array}\right) .
\end{aligned}
$$

A manifold whose curvature tensor is of the form

$$
R_{i j k l}=\kappa\left(g_{i l} g_{j k}-g_{j l} g_{i k}\right)
$$

is called a manifold of constant curvature [7]. Here $\kappa$ is the sectional curvature of the manifold.

From (3.2) and the above definition, we write

$$
\begin{gathered}
R_{\text {siaj }}=\kappa\left(g_{s j} g_{i a}-g_{i j} g_{s a}\right) \\
R_{s j a i}=\kappa\left(g_{s i} g_{j a}-g_{j i} g_{s a}\right) \\
\Rightarrow \quad R_{s i a j}-R_{s j a i}=\kappa\left(g_{s j} g_{i a}-g_{s i} g_{j a}\right) .
\end{gathered}
$$

When we use the above equation (3.4) on the equation (3.2) and take $\kappa=1$, we obtain

$$
\begin{aligned}
& y^{s}\left[R_{\text {siaj }}-R_{s j a i}+g_{s i} g_{j a}-g_{s j} g_{i a}\right] v^{a} \\
= & y^{s}\left[\kappa\left(g_{s j} g_{i a}-g_{s i} g_{j a}\right)+g_{s i} g_{j a}-g_{s j} g_{i a}\right] v^{a} \\
= & y^{s}\left[\left(g_{s j} g_{i a}-g_{s i} g_{j a}\right)+g_{s i} g_{j a}-g_{s j} g_{i a}\right] v^{a} \\
= & 0 .
\end{aligned}
$$

Hence, as a corollary of Lemma 3.1, we obtain

Proposition 3.2. Let $M$ be a (pseudo-)Riemannian manifold and TM be its tangent bundle with the semi-symmetric metric connection $\bar{\nabla}$. Then, for any vector field $V$ on $M$,

i) The vertical lift ${ }^{V} V$ is a harmonic vector field on $T M$ with respect to the semi-symmetric metric connection $\bar{\nabla}$ if and only if the vector field $V$ is a harmonic vector field with respect to the Levi-Civita connection $\nabla$;

ii) The complete lift ${ }^{C} V$ is a harmonic vector field on $T M$ with respect to the semi-symmetric metric connection $\bar{\nabla}$ if and only if the vector field $V$ is a harmonic vector field with respect to the Levi-Civita connection $\nabla$ and $g_{s i} g_{j a}-g_{s j} g_{i a}=0$;

iii) The horizontal lift ${ }^{H} V$ is a harmonic vector field on $T M$ with respect to the semi-symmetric metric connection $\bar{\nabla}$ if and only if the vector field $V$ is parallel with respect to the Levi-Civita connection $\nabla$ and $M$ has constant sectional curvature 1. 
Some Vector Fields on the Tangent Bundle with a Semi-symmetric Metric Connection675

\subsection{Concurrent vector fields}

Definition 3.3. A vector field $\widetilde{V}=v^{h} E_{h}+v^{\bar{h}} E_{\bar{h}}$ on $T M$ is called a concurrent vector field with respect to the semi-symmetric metric connection $\bar{\nabla}$ if it satisfies

$$
\bar{\nabla}_{\beta} \widetilde{V}^{\epsilon}=\bar{\nabla}_{E_{\beta}} \widetilde{V}^{\epsilon}=\widetilde{k} \delta_{\beta}^{\epsilon},
$$

where $\widetilde{k}$ is a function on $T M$ and $\delta_{\beta}^{\epsilon}$ is the Kronecker symbol.

Proposition 3.3. Let $M$ be a (pseudo-)Riemannian manifold and TM be its tangent bundle with the semi-symmetric metric connection $\bar{\nabla}$. The vector field $\widetilde{V}$ on $T M$ is concurrent with respect to semi-symmetric metric connection $\bar{\nabla}$ if and only if the vector field $\widetilde{V}$ has the form

$$
\widetilde{V}=\left(\begin{array}{c}
v^{h} \\
\frac{1}{n}[\operatorname{trace}(\nabla V)] y^{h}
\end{array}\right)
$$

and the following condition is satisfied

$$
\frac{1}{n}\left[\nabla_{j}(\operatorname{trace}(\nabla V)) y^{h}\right]+\left(y^{s} R_{s j a}^{h}+y_{a} \delta_{j}^{h}-y^{h} g_{j a}\right) v^{a}=0 .
$$

Proof. With respect to the adapted frame, firstly putting $\epsilon=h, \beta=\bar{j}$ in (3.5), it follows that

$$
\begin{aligned}
\bar{\nabla}_{\bar{j}} v^{h} & =E_{\bar{j}} v^{h}+\bar{\Gamma}_{\bar{j} a}^{h} v^{a}+\bar{\Gamma}_{\bar{j} \bar{a}}^{h} v^{\bar{a}}=\widetilde{k} \delta_{\bar{j}}^{h} \\
& \Rightarrow \partial_{\bar{j}} v^{h}=0 \\
& \Rightarrow v^{h}=v^{h}\left(x^{h}\right)
\end{aligned}
$$

Similarly putting $\epsilon=h, \beta=j$ and $\epsilon=\bar{h}, \beta=\bar{j}$, we respectively get

$$
\begin{aligned}
\bar{\nabla}_{j} v^{h} & =E_{j} v^{h}+\bar{\Gamma}_{j a}^{h} v^{a}+\bar{\Gamma}_{j \bar{a}}^{h} v^{\bar{a}}=\widetilde{k} \cdot \delta_{j}^{h} \\
& \Rightarrow \partial_{j} v^{h}+\Gamma_{j a}^{h} v^{a}=\widetilde{k} \cdot \delta_{j}^{h} \\
& \Rightarrow \nabla_{j} v^{h}=\widetilde{k} \cdot \delta_{j}^{h} \quad(h \rightarrow j) \\
& \Rightarrow \frac{1}{n} \nabla_{j} v^{j}=\widetilde{k}
\end{aligned}
$$

and

$$
\begin{aligned}
\bar{\nabla}_{\bar{j}} v^{\bar{h}} & =E_{\bar{j}} v^{\bar{h}}+\bar{\Gamma}_{\bar{j} a}^{\bar{h}} v^{a}+\bar{\Gamma}_{\bar{j} \bar{a}}^{\bar{h}} v^{\bar{a}}=\widetilde{k} \cdot \delta_{\bar{j}}^{\bar{h}} \\
& \Rightarrow \quad \partial_{\bar{j}} v^{\bar{h}}=\frac{1}{n} \nabla_{j} v^{j} \cdot \delta_{\bar{j}}^{\bar{h}} \\
& \Rightarrow \quad \partial_{\bar{j}} v^{\bar{h}}=\frac{1}{n}\left[\operatorname{trace}(\nabla V) \delta_{\bar{j}}\right] \\
& \Rightarrow \quad \partial_{\bar{j}} v^{\bar{h}}=\frac{1}{n}\left[\operatorname{trace}(\nabla V)\left(\partial_{\bar{j}} y^{h}\right)\right]
\end{aligned}
$$




$$
\begin{aligned}
& \Rightarrow \quad \partial_{\bar{j}} v^{\bar{h}}=\partial_{\bar{j}}\left[\frac{1}{n} \operatorname{trace}(\nabla V) y^{h}\right] \\
& \Rightarrow v^{\bar{h}}=\frac{1}{n}[\operatorname{trace}(\nabla V)] y^{h} .
\end{aligned}
$$

Finally putting $\epsilon=\bar{h}, \beta=j$, we find

$$
\begin{aligned}
& \bar{\nabla}_{j} v^{\bar{h}}=E_{j} v^{\bar{h}}+\bar{\Gamma}_{j a}^{\bar{h}} v^{a}+\bar{\Gamma}_{j \bar{a}}^{\bar{h}} v^{\bar{a}}=\widetilde{k} \delta_{j}^{\bar{h}} \\
& \Rightarrow \quad E_{j}\left[\frac{1}{n}[\operatorname{trace}(\nabla V)] y^{h}\right]+\left(y^{s} R_{s j a}^{h}+y_{a} \delta_{j}^{h}-y^{h} g_{j a}\right) v^{a} \\
& +\Gamma_{j a}^{h}\left[\frac{1}{n}[\operatorname{trace}(\nabla V)] y^{a}\right]=0 \\
& \begin{array}{c}
\left(\partial_{j}-y^{s} \Gamma_{s j}^{m} \partial_{\bar{m}}\right)\left[\frac{1}{n}[\operatorname{trace}(\nabla V)] y^{h}\right] \\
\Rightarrow \quad+\left(y^{s} R_{s j a}^{h}+y_{a} \delta_{j}^{h}-y^{h} g_{j a}\right) v^{a}+y^{a} \Gamma_{j a}^{h}\left[\frac{1}{n}[\operatorname{trace}(\nabla V)]\right]=0
\end{array} \\
& \begin{array}{c}
\frac{1}{n}\left[\partial_{j}(\operatorname{trace}(\nabla V)) y^{h}\right]-y^{s} \Gamma_{s j}^{h}\left[\frac{1}{n}[\operatorname{trace}(\nabla V)]\right] \\
\Rightarrow \quad+\left(y^{s} R_{s j a}^{h}+y_{a} \delta_{j}^{h}-y^{h} g_{j a}\right) v^{a}+y^{a} \Gamma_{j a}^{h}\left[\frac{1}{n}[\operatorname{trace}(\nabla V)]\right]=0
\end{array} \\
& \Rightarrow \frac{1}{n}\left[\partial_{j}(\operatorname{trace}(\nabla V)) y^{h}\right]+\left(y^{s} R_{s j a}^{h}+y_{a} \delta_{j}^{h}-y^{h} g_{j a}\right) v^{a}=0 \\
& \Rightarrow \frac{1}{n}\left[\nabla_{j}(\operatorname{trace}(\nabla V)) y^{h}\right]+\left(y^{s} R_{s j a}^{h}+y_{a} \delta_{j}^{h}-y^{h} g_{j a}\right) v^{a}=0 \text {. }
\end{aligned}
$$

\subsection{Conformal vector fields}

Let $\widetilde{V}$ be a vector field on $T M$ with components $\left(v^{h}, v^{\bar{h}}\right)$ with respect to the adapted frame $\left\{E_{\beta}\right\}$. Then $\widetilde{V}$ is a fibre-preserving vector field on $T M$ if and only if $v^{h}$ depends only on the variables $\left(x^{h}\right)$.

Definition 3.4. A vector field $\widetilde{V}=v^{h} E_{h}+v^{\bar{h}} E_{\bar{h}}$ on $T M$ is called a fibrepreserving conformal vector field with respect to the semi-symmetric metric connection $\bar{\nabla}$ if it satisfies

$$
L_{\widetilde{V}}^{C} g_{\alpha \beta}=\left(\bar{\nabla}_{\alpha} \widetilde{V}^{\epsilon}\right)^{C} g_{\in \beta}+\left(\bar{\nabla}_{\beta} \widetilde{V}^{\epsilon}\right)^{C} g_{\in \alpha}=2 \widetilde{\Omega}^{C} g_{\alpha \beta} .
$$

Putting $(\alpha, \beta)=(i, \bar{j}),(\bar{i}, j)$ and $(i, j)$, from the above equation, it can be written the following system

$$
\left\{\begin{array}{c}
i)\left(\nabla_{i} v^{h}\right) g_{h j}+\left(E_{\bar{j}} v^{\bar{h}}\right) g_{h i}=2 \widetilde{\Omega} g_{i j} \\
i i)\left(E_{\bar{i}} \bar{v}^{\bar{h}}\right) g_{h j}+\left(\nabla_{j} v^{h}\right) g_{h i}=2 \widetilde{\Omega} g_{i j}, \\
i i i) \begin{array}{c}
{\left[E_{i} v^{\bar{h}}+\left(y^{s} R_{s i a}{ }^{h}+y_{a} \delta_{i}^{h}-y^{h} g_{i a}\right) v^{a}+\Gamma_{i a}^{h} v^{\bar{a}}\right] g_{h j}} \\
+\left[E_{j} v^{\bar{h}}+\left(y^{s} R_{s j a}{ }^{h}+y_{a} \delta_{j}^{h}-y^{h} g_{j a}\right) v^{a}+\Gamma_{j a}^{h} v^{\bar{a}}\right] g_{h i}
\end{array}=0 .
\end{array}\right.
$$

Proposition 3.4. The scalar function $\widetilde{\Omega}$ on $T M$ depends only on the variables $\left(x^{h}\right)$ with respect to the induced coordinates $\left(x^{h}, y^{h}\right)$. 
Some Vector Fields on the Tangent Bundle with a Semi-symmetric Metric Connection677

Proof. Applying $E_{\bar{k}}$ to the both sides of the equation (ii) in (3.6), we have

$$
g_{h j} E_{\bar{k}} E_{\bar{i}} v^{\bar{h}}=2 E_{\bar{k}}(\bar{\Omega}) g_{i j}
$$

from which we get

$$
E_{\bar{k}}(\bar{\Omega}) g_{i j}=E_{\bar{i}}(\bar{\Omega}) g_{k j}
$$

It follows that

$$
(n-1) E_{\bar{k}}(\bar{\Omega})=0 .
$$

This shows that the scalar function $\widetilde{\Omega}$ on $T M$ depends only on the variables $\left(x^{h}\right)$ with respect to the induced coordinates $\left(x^{h}, y^{h}\right)$. Thus we can regard $\widetilde{\Omega}$ as a function on $M$ and in the following we write $\rho$ instead of $\widetilde{\Omega}$.

From (3.6) and Proposition 3.4, $E_{\bar{i}}\left(v^{\bar{h}}\right)$ depends only the variables $\left(x^{h}\right)$, thus we can put

$$
v^{\bar{h}}=y^{a} A_{a}^{h}+B^{h},
$$

where $A_{a}^{h}$ and $B^{h}$ are certain functions which depend only on the variable $\left(x^{h}\right)$. Furthermore, we can easily show that $A_{a}^{h}$ and $B^{h}$ are the components of a $(1,1)$ tensor field and a contravariant vector field on $M$, respectively.

Any vector field $V$ on a (pseudo-)Riemannian manifold $(M, g)$ is a Killing vector field if $L_{V} g_{i j}=\nabla_{i} v_{j}+\nabla_{j} v_{i}=0$.

Proposition 3.5. If we put

$$
B=B^{h} \frac{\partial}{\partial x^{h}},
$$

then the vector field $B$ on $M$ is a Killing vector field with respect to the Levi-Civita connection $\nabla$.

Proof. Substituting (3.7) into the equation (iii) in (3.6) we have

$$
\nabla_{i} B_{j}+\nabla_{j} B_{i}=0
$$

and

$$
\begin{aligned}
& v^{a}\left(R_{s i a j}+R_{s j a i}+g_{s a} g_{i j}-g_{i a} g_{s j}+g_{s a} g_{j i}-g_{j a} g_{s i}\right) \\
& +\nabla_{i} A_{s j}+\nabla_{j} A_{s i}=0
\end{aligned}
$$

where $B_{i}=g_{i m} B^{m}$ and $A_{s j}=g_{h j} A_{s}^{h}$. Hence by (3.8), it follows

$$
L_{B} g_{i j}=\nabla_{i} B_{j}+\nabla_{j} B_{i}=0 .
$$

This shows $B$ is a Killing vector field on $M$ with respect to the Levi-Civita connection $\nabla$. 
Substituting (3.7) into the equation (ii) in (3.6), we have

$$
\begin{aligned}
& E_{\bar{i}}\left(v^{\bar{h}}\right) g_{h j}+\left(\nabla_{j} v^{h}\right) g_{h i}=2 \rho g_{i j} \\
\Rightarrow & \partial_{\bar{i}}\left(y^{s} A_{s}^{h}+B^{h}\right) g_{h j}+\left(\nabla_{j} v^{h}\right) g_{h i}=2 \rho g_{i j} \\
\Rightarrow & A_{i}^{h} g_{h j}+\left(\nabla_{j} v^{h}\right) g_{h i}=2 \rho g_{i j} \\
\Rightarrow & g_{h j} A_{i}^{h}=2 \rho g_{i j}-g_{h i}\left(\nabla_{j} v^{h}\right) .
\end{aligned}
$$

Let $\nabla$ be a linear connection on $M$. A vector field $V$ on $M$ is said to be a projective vector field if there exists a 1 -form $\theta$ such that

$$
\left(L_{V} \nabla\right)(X, Y)=\theta(X) Y+\theta(Y) X
$$

for any vector fields $X$ and $Y$ on $M$. In this case $\theta$ is called the associated 1-form of $V$. It can locally be expressed in the following form

$$
L_{V} \Gamma_{i j}^{h}=\theta_{i} \delta_{j}^{h}+\theta_{j} \delta_{i}^{h} .
$$

Proposition 3.6. The vector field $V$ with components $\left(v^{h}\right)$ is a projective vector field on $M$ with respect to the Levi-Civita connection $\nabla$, if $\delta_{a}^{h} g_{i j}-g_{i a} \delta_{j}^{h}+\delta_{a}^{h} g_{j i}-$ $g_{j a} \delta_{i}^{h}=0$.

Proof. Applying the covariant derivative $\nabla_{k}$ to the both sides of (3.10), we obtain

$$
\begin{aligned}
g_{h j} \nabla_{k} A_{i}^{h} & =\nabla_{k}\left[2 \rho g_{i j}-g_{h i}\left(\nabla_{j} v^{h}\right)\right] \\
& =2\left(\nabla_{k} \rho\right) g_{i j}-g_{h i} \nabla_{k} \nabla_{j} v^{h} \\
& =2 \rho_{k} g_{i j}-g_{h i}\left(L_{V} \Gamma_{k j}^{h}-R_{a k j}^{h} v^{a}\right) \\
\nabla_{k} A_{i j} & =2 \rho_{k} g_{i j}-L_{V} \Gamma_{k j}^{h} g_{h i}-R_{a k i j} v^{a} .
\end{aligned}
$$

Substituting (3.11) into (3.9), we have

$$
\begin{gathered}
v^{a}\left(R_{s i a j}+R_{s j a i}+g_{s a} g_{i j}-g_{i a} g_{s j}+g_{s a} g_{j i}-g_{j a} g_{s i}\right)+\nabla_{i} A_{s j}+\nabla_{j} A_{s i}=0 \\
v^{a}\left(R_{s i a j}+R_{s j a i}+g_{s a} g_{i j}-g_{i a} g_{s j}+g_{s a} g_{j i}-g_{j a} g_{s i}\right) \\
+2 \rho_{i} g_{s j}-L_{V} \Gamma_{i j}^{h} g_{h s}-R_{a i s j} v^{a}+2 \rho_{j} g_{s i}-L_{V} \Gamma_{j i}^{h} g_{h s}-R_{a j s i} v^{a}=0 \\
v^{a}\left(g_{s a} g_{i j}-g_{i a} g_{s j}+g_{s a} g_{j i}-g_{j a} g_{s i}\right)+2\left(\rho_{i} g_{s j}+\rho_{j} g_{s i}\right)=2 L_{V} \Gamma_{i j}^{h} g_{h s} \\
L_{V} \Gamma_{i j}^{h}=\rho_{i} \delta_{j}^{h}+\rho_{j} \delta_{i}^{h}+\frac{1}{2} v^{a}\left(\delta_{a}^{h} g_{i j}-g_{i a} \delta_{j}^{h}+\delta_{a}^{h} g_{j i}-g_{j a} \delta_{i}^{h}\right),
\end{gathered}
$$

where $\rho_{i}=\nabla_{i} \rho$. Hence, $V$ is a projective vector field on $M$ with respect to the Levi-Civita connection $\nabla$. 
Now we consider the converse problem, that is, let $M$ admit a projective vector field $V=v^{h} \frac{\partial}{\partial x^{h}}$ with respect to the Levi-Civita connection $\nabla$. Then we have the following proposition.

Proposition 3.7. The vector field $\widetilde{V}$ on $T M$ defined by

$$
\widetilde{V}=v^{h} E_{h}+\left(y^{s} A_{s}^{h}+B^{h}\right) E_{\bar{h}}
$$

is a fibre-preserving conformal vector field on $T M$ with respect to the semi-symmetric metric connection $\bar{\nabla}$, where $A_{i}^{h}=g^{h a} A_{a i}, A_{i j}=2 \rho g_{i j}-\nabla_{j} v_{i}$, and $g_{j i} B^{j}=B_{i}$, $2 p_{i} g_{s j}-L_{V} \Gamma_{i j}^{h} g_{h s}+\left(g_{s m} g_{i j}-g_{i m} g_{s j}\right)=0$.

Proof. If $B_{h}, v^{h}$ and $A_{i}^{h}$ are given so that they satisfy the above assumptions, we see that $\widetilde{V}=v^{h} E_{h}+\left(y^{s} A_{s}^{h}+B^{h}\right) E_{\bar{h}}$ is a fibre-preserving conformal vector field on $T M$ with respect to the semi-symmetric metric connection $\bar{\nabla}$. We omit standard calculations.

\subsection{Projective vector fields}

In this section, we study fibre-preserving projective vector fields on $T M$ with respect to the semi-symmetric metric connection $\bar{\nabla}$. We shall first state following lemma which is needed later on.

Lemma 3.2. The Lie derivations of the adapted frame with respect to the fibrepreserving vector field $\widetilde{V}=v^{h} E_{h}+v^{\bar{h}} E_{\bar{h}}$ are given as follows

$$
\begin{gathered}
L_{\widetilde{V}} E_{h}=-\left(\partial_{h} v^{a}\right) E_{a}+\left\{y^{b} v^{c} R_{h c b}^{a}-v^{\bar{b}} \Gamma_{b h}^{a}-\left(E_{h} v^{\bar{a}}\right\} E_{\bar{a}},\right. \\
L_{\widetilde{V}} E_{\bar{h}}=\left\{v^{b} \Gamma_{b h}^{a}-\left(E_{\bar{h}} v^{\bar{a}}\right)\right\} E_{\bar{a}} .
\end{gathered}
$$

The general form of fibre-preserving vector fields on $T M$ with respect to the semi-symmetric metric connection $\bar{\nabla}$ are given by

Theorem 3.1. Let $M$ be a (pseudo-)Riemannian manifold and $T M$ be its tangent bundle with the semi-symmetric metric connection $\bar{\nabla}$. Then a vector field $\widetilde{V}$ is a fibre-preserving projective vector field with associated 1-form $\bar{\theta}$ on $T M$ with respect to the semi-symmetric metric connection $\bar{\nabla}$ if and only if the vector field $\widetilde{V}$ has the following form

$$
\widetilde{V}={ }^{H} V+{ }^{V} B+\gamma A
$$


where the vector fields $V=\left(v^{h}\right), B=\left(B^{h}\right)$, the $(1,1)$-tensor field $A=\left(A_{i}^{h}\right)$ and the associated 1 -form $\bar{\theta}$ satisfy the following conditions

$$
\begin{gathered}
(i) \bar{\theta}=\theta_{i} d x^{i}, \\
(i i) \nabla_{i} \theta_{j}=(n-1)\left(L_{V} g_{i j}\right), \\
(i i i) \nabla_{j} A_{i}^{h}=\theta_{j} \delta_{i}^{h}-v^{c} R_{c j i}^{h}, \\
(i v) \nabla_{i} \nabla_{j} v^{h}+R_{a i j}^{h} v^{a}=\theta_{i} \delta_{j}^{h}+\theta_{j} \delta_{i}^{h}, \\
(v) \nabla_{i} \nabla_{j} B^{k}+R_{h i j}^{k} B^{h}+B^{h} g_{h j} \delta_{i}^{k}-B^{k} g_{i j}=0, \\
(v i) L_{V} \Gamma_{i j}^{h}=\theta_{i} \delta_{j}^{h}+\theta_{j} \delta_{i}^{h} .
\end{gathered}
$$

Proof. A vector field $\widetilde{V}=v^{h} E_{h}+v^{\bar{h}} E_{\bar{h}}$ on $T M$ is a fibre-preserving projective vector field with respect to the semi-symmetric metric connection $\bar{\nabla}$ if and only if there exists a 1 -form $\widetilde{\theta}$ with components $\left(\widetilde{\theta}_{i}, \widetilde{\theta}_{\bar{i}}\right)$ on $T M$ such that

$$
\begin{aligned}
\left(L_{\widetilde{X}} \bar{\nabla}\right)(\widetilde{Y}, \widetilde{Z}) & =L_{\widetilde{X}}\left(\bar{\nabla}_{\widetilde{Y}} \widetilde{Z}\right)-\bar{\nabla}_{\widetilde{Y}}\left(L_{\widetilde{X}} \widetilde{Z}\right)-\bar{\nabla}_{\left(L_{\widetilde{X}} \widetilde{Y}\right)} \widetilde{Z} \\
& =\widetilde{\theta}(\widetilde{Y}) \widetilde{Z}+\widetilde{\theta}(\widetilde{Z}) \widetilde{Y}
\end{aligned}
$$

for any vector fields $\widetilde{Y}$ and $\widetilde{Z}$ on $T M$. We compute the following system

$$
\begin{aligned}
\left(L_{\widetilde{V}} \bar{\nabla}\right)\left(E_{\bar{i}}, E_{\bar{j}}\right) & =L_{\widetilde{V}}\left(\bar{\nabla}_{E_{\bar{i}}} E_{\bar{j}}\right)-\bar{\nabla}_{E_{\bar{i}}}\left(L_{\widetilde{V}} E_{\bar{j}}\right)-\bar{\nabla}_{\left(L_{\tilde{V}} E_{\bar{i}}\right)} E_{\bar{j}} \\
& =\widetilde{\theta}\left(E_{\bar{i}}\right) E_{\bar{j}}+\widetilde{\theta}\left(E_{\bar{j}}\right) E_{\bar{i}}, \\
\left(L_{\widetilde{V}} \bar{\nabla}\right)\left(E_{\bar{i}}, E_{j}\right) & =L_{\widetilde{V}}\left(\bar{\nabla}_{E_{\bar{i}}} E_{j}\right)-\bar{\nabla}_{E_{\bar{i}}}\left(L_{\widetilde{V}} E_{j}\right)-\bar{\nabla}_{\left(L_{\tilde{V}} E_{\bar{i}}\right)} E_{j} \\
& =\widetilde{\theta}\left(E_{\bar{i}}\right) E_{j}+\widetilde{\theta}\left(E_{j}\right) E_{\bar{i}}, \\
\left(L_{\widetilde{V}} \bar{\nabla}\right)\left(E_{i}, E_{j}\right) & =L_{\widetilde{V}}\left(\bar{\nabla}_{E_{i}} E_{j}\right)-\bar{\nabla}_{E_{i}}\left(L_{\widetilde{V}} E_{j}\right)-\bar{\nabla}_{\left(L_{\tilde{V}} E_{i}\right)} E_{j} \\
& =\widetilde{\theta}\left(E_{i}\right) E_{j}+\widetilde{\theta}\left(E_{j}\right) E_{i} .
\end{aligned}
$$

From (3.12), by virtue of (2.2) and Lemma 3.2 we obtain

$$
\left\{\partial_{\bar{i}}\left(\partial_{\bar{j}} v^{\bar{a}}\right\} E_{\bar{a}}=\widetilde{\theta}_{\bar{i}} E_{\bar{j}}+\widetilde{\theta}_{\bar{j}} E_{\bar{i}} .\right.
$$

Similarly, from (3.13) we get

$$
\left\{-v^{c} R_{j c i}^{a}+\left(E_{\bar{i}} v^{\bar{b}}\right) \Gamma_{b j}^{a}+E_{\bar{i}}\left(E_{j} v^{\bar{a}}\right)\right\} E_{\bar{a}}=\widetilde{\theta}_{\bar{i}} E_{j}+\widetilde{\theta}_{j} E_{\bar{i}}
$$

from which, we have

$$
\widetilde{\theta}_{\bar{i}}=0 .
$$

Due to $\widetilde{\theta}_{\bar{i}}=0,(3.15)$ to

$$
\partial_{\bar{i}}\left(\partial_{\bar{j}} v^{\bar{a}}\right)=0,
$$


Some Vector Fields on the Tangent Bundle with a Semi-symmetric Metric Connection681

and we obtain

$$
v^{\bar{a}}=y^{s} A_{s}^{a}+B^{a},
$$

where $A_{s}^{a}$ and $B^{a}$ are certain functions which depend only on the variables $\left(x^{h}\right)$ and the coordinate transformation rule implies that $A$ is a $(1,1)$-tensor field with components $\left(A_{s}^{a}\right)$ and $B$ is a vector field with components $\left(B^{a}\right)$. Hence, the fibrepreserving projective vector field $\widetilde{V}$ on $T M$ can be expressed in the following form

$$
\begin{aligned}
\widetilde{V} & =v^{h} E_{h}+v^{\bar{h}} E_{\bar{h}}=v^{h} E_{h}+\left\{y^{s} A_{s}^{a}+B^{a}\right\} E_{\bar{h}} \\
& ={ }^{H} V+{ }^{V} B+\gamma A .
\end{aligned}
$$

Substituting (3.18) into (3.16), we obtain

$$
R_{a j i}^{h} v^{a}+\nabla_{j} A_{i}^{h}=\delta_{i}^{h} \theta_{j} .
$$

Substituting (3.18) and (3.20) into (3.14), we have

$$
\begin{aligned}
& \left\{\nabla_{i} \nabla_{j} v^{h}+R_{a i j}^{h} v^{a}\right\} E_{h}+\left\{\nabla_{i} \nabla_{j} B^{k}+R_{h i j}^{k} B^{h}+B^{h} g_{h j} \delta_{i}^{k}\right. \\
& -B^{k} g_{i j}+y^{s}\left(\nabla_{i} \nabla_{j} A_{s}^{k}+A_{s}^{h} R_{h i j}^{k}-R_{s i j}^{a} A_{a}^{k}+v^{h} \nabla_{h} R_{s i j}^{k}\right. \\
& -v^{h} \nabla_{i} R_{j h s}^{k}+\nabla_{j} v^{h} R_{s i h}^{k}+\nabla_{i} v^{h} R_{s j h}^{k}+\nabla_{j} v^{a} g_{s a} \delta_{i}^{k} \\
& \left.\left.-\nabla_{j} v^{a} \delta_{s}^{k} g_{i a}+\nabla_{i} v^{a} g_{s j} \delta_{a}^{k}-\nabla_{i} v^{a} \delta_{s}^{k} g_{a j}+A_{s}^{h} g_{h j} \delta_{i}^{k}-g_{s j} A_{i}^{k}\right)\right\} E_{\bar{h}} \\
= & \widetilde{\theta}_{i} E_{j}+\widetilde{\theta}_{j} E_{i} .
\end{aligned}
$$

From (3.21), we have

$$
\begin{gathered}
\nabla_{i} \nabla_{j} v^{h}+R_{a i j}{ }^{h} v^{a}=\widetilde{\theta}_{i} \delta_{j}^{h}+\widetilde{\theta}_{j} \delta_{i}^{h}, \\
\nabla_{i} \nabla_{j} B^{k}+R_{h i j}{ }^{k} B^{h}+B^{h} g_{h j} \delta_{i}^{k}-B^{k} g_{i j}=0, \\
\nabla_{i} \nabla_{j} A_{s}^{k}+A_{s}^{h} R_{h i j}^{k}-R_{s i j} A_{a}^{k}+v^{h} \nabla_{h} R_{s i j}{ }^{k} \\
-v^{h} \nabla_{i} R_{j h s}{ }^{k}+\nabla_{j} v^{h} R_{s i h}{ }^{k}+\nabla_{i} v^{h} R_{s j h}^{k} \\
+\nabla_{j} v^{a} g_{s a} \delta_{i}^{k}-\nabla_{j} v^{a} \delta_{s}^{k} g_{i a}+\nabla_{i} v^{a} g_{s j} \delta_{a}^{k} \\
-\nabla_{i} v^{a} \delta_{s}^{k} g_{a j}+A_{s}^{h} g_{h j} \delta_{i}^{k}-g_{s j} A_{i}^{k} \\
=0 .
\end{gathered}
$$

The equation (3.22) shows that the induced vector field $V=v^{h} \frac{\partial}{\partial x^{h}}$ is a projective vector field with respect to the Levi-Civita Connection $\nabla$. Hence we obtain

$$
L_{V} R_{i j}=-(n-1) \nabla_{i} \theta_{j}
$$

Contracting $k$ and $s$ in (3.24) and using (3.20) and (3.25), we get

$$
\nabla_{i} \theta_{j}=(n-1)\left(L_{V} g\right)_{i j}
$$


In the case, (3.24) is reduced to

$$
\begin{aligned}
& A_{s}^{h} R_{h i j}{ }^{k}-R_{s i j}{ }^{a} A_{a}^{k}+v^{h} \nabla_{h} R_{s i j}{ }^{k}+\nabla_{j} v^{h} R_{s i h}^{k} \\
& +\nabla_{i} v^{h} R_{s h j}^{k}+\nabla_{j} v_{s} \delta_{i}^{k}+\nabla_{i} v^{k} g_{s j}+A_{s j} \delta_{i}^{k}-g_{s j} A_{i}^{k} \\
= & 0 .
\end{aligned}
$$

Conversely, if $B^{h}, v^{h}, \theta_{h}$ and $A_{i}^{h}$ are given so that they satisfy (i)-(vi), reserving the above steps, we see that $\widetilde{X}={ }^{H} V+{ }^{V} B+\gamma A$ is a fibre-preserving projective vector field on $T M$ with respect to the semi-symmetric metric connection $\bar{\nabla}$. Hence, the proof is complete.

Let $\widetilde{V}$ be a fibre-preserving vector field on $T M$ with components $\left(v^{h}, v^{\bar{h}}\right)$. It is well-known that every fibre-preserving vector field $\widetilde{V}$ on $T M$ induces a vector field $V$ on $M$ with components $\left(v^{h}\right)$. The below result follows immediately from Theorem 3.1 and from its Proof.

Corollary 3.1. Let $M$ be a (pseudo-)Riemannian manifold and TM be its tangent bundle with the semi-symmetric metric connection $\bar{\nabla}$. Every fibre-preserving projective vector field $\widetilde{V}$ is of the form (3.19) and it naturally induces a projective vector field $V$ on $M$.

Let $\widetilde{V}$ be a vector field on $T M$ with components $\left(v^{h}, v^{\bar{h}}\right)$ with respect to the adapted frame $\left\{E_{\beta}\right\}$. Then $\widetilde{V}$ is a vertical vector field on $T M$ if and only if $v^{h}=0$. In the present case, the vector field $\widetilde{V}$ in Theorem 3.1 reduces to $\widetilde{V}={ }^{V} B+\gamma A$. Hence, from the Theorem 3.1, we obtain the following conclusion.

Corollary 3.2. Let $M$ be a (pseudo-)Riemannian manifold and TM be its tangent bundle with the semi-symmetric metric connection $\bar{\nabla}$. If TM admits a vertical projective vector field $\widetilde{V}$, then the vector field $\widetilde{V}$ is defined by

$$
\widetilde{V}={ }^{V} B+\gamma A,
$$

where the vector field $B=\left(B^{h}\right)$, the $(1,1)$-tensor field $A=\left(A_{i}^{h}\right)$ and the associated 1 -form $\widetilde{\theta}$ satisfy the following conditions

$$
\begin{gathered}
(i) \bar{\theta}=\theta_{i} d x^{i}, \\
(i i) \nabla_{j} A_{i}^{h}=\theta_{j} \delta_{i}^{h}, \\
(i i i) \nabla_{i} \theta_{j}=0, \\
(i v) \nabla_{i} \nabla_{j} B^{k}+R_{h i j}^{k} B^{h}+B^{h} g_{h j} \delta_{i}^{k}-B^{k} g_{i j}=0, \\
(v) A_{s}^{h} R_{h i j}^{k}-R_{s i j} A_{a}^{k}+A_{s j} \delta_{i}^{k}-g_{s j} A_{i}^{k}=0 .
\end{gathered}
$$


Some Vector Fields on the Tangent Bundle with a Semi-symmetric Metric Connection683

\section{R E F E R E N C E S}

1. A. Friedmann and J.A. Schouten: Uber die geometrie der halbsymmetrischen ubertragungen. Math. Z. 21 (1924), no.1, 211-223.

2. H.A. Hayden: Sub-spaces of a space with torsion. Proc. London Math. Soc. S234 (1932), 27-50.

3. T. IMAI: Notes on semi-symmetric metric connections. Commemoration volumes for Prof. Dr. Akitsugu Kawaguchi's seventieth birthday. Tensor (N.S.) bf 24 (1972), 293-296.

4. T. IMAI: Hypersurfaces of a Riemannian manifold with semi-symmetric metric connection. Tensor (N.S.) 23 (1972), 300-306.

5. S. SASAKI: On the differential geometry of tangent bundles of Riemannian manifolds. Tohoku Math. J. 10 (1958), no.3, 338-354.

6. K. Yano: On semi-symmetric metric connection. Rev. Roumaine Math. Pures Appl. 15 (1970), 1579-1586.

7. K. Yano: Differential Geometry on Complex and Almost Complex Spaces. The Macmillan Company, New York, 1965.

8. K. YANO and S. IshinARA: Tangent and Cotangent Bundles. Marcel Dekker Inc., New York, 1973.

9. A. Gezer and E. KARAKAs: On a semi-symmetric metric connection on the tangent bundle with the complete lift metric. Riv. Mat. Univ. Parma (N.S) 9 (2018), no.1, 73-84. 